\title{
Pressure Dependence of Fragile-to-Strong Transition and a Possible Second Critical Point in Supercooled Confined Water
}

\author{
Li Liu, ${ }^{1}$ Sow-Hsin Chen, ${ }^{1, *}$ Antonio Faraone, ${ }^{1,2}$ Chun-Wan Yen, ${ }^{3}$ and Chung-Yuan $\mathrm{Mou}^{3}$ \\ ${ }^{1}$ Department of Nuclear Science and Engineering, Massachusetts Institute of Technology, Cambridge, Massachusetts 02139, USA \\ ${ }^{2}$ Department of Material Science and Engineering, University of Maryland, College Park, MD 20742, USA \\ and NIST Center for Neutron Research, Gaithersburg MD, 20899-8562, USA \\ ${ }^{3}$ Department of Chemistry, National Taiwan University, Taipei 106, Taiwan, Republic of China
}

(Received 12 June 2005; published 9 September 2005)

\begin{abstract}
By confining water in nanopores of silica glass, we can bypass the crystallization and study the pressure effect on the dynamical behavior in deeply supercooled state using neutron scattering. We observe a clear evidence of a cusplike fragile-to-strong (FS) dynamic transition. Here we show that the transition temperature decreases steadily with an increasing pressure, until it intersects the homogenous nucleation temperature line of bulk water at a pressure of 1600 bar. Above this pressure, it is no longer possible to discern the characteristic feature of the FS transition. Identification of this end point with the possible second critical point is discussed.
\end{abstract}

DOI: $10.1103 /$ PhysRevLett.95.117802

PACS numbers: 61.20.Lc, 61.12.Ex, 61.20.Ja

Water is a continuing source of fascination to scientists because of its many counterintuitive low-temperature properties. Although the stable form of water at sufficiently low temperature is inevitably crystalline, liquid water can also exist in a metastable supercooled state far below the melting point. One of the most intriguing questions related to the unusual properties of supercooled water is whether two critical points may exist in a single component liquid [1].

It has been known since 1970s that a number of thermodynamic response functions of supercooled water, notably the isothermal compressibility $K_{T}$ and the constantpressure specific heat $C_{P}$, show a power-law divergence behavior at a singular temperature, experimentally determined to be $228 \mathrm{~K}$ at the ambient pressure [2,3]. Concurrently, the transport properties, such as the shear viscosity $\eta$ and the inverse self-diffusion constant $D$, diverge according to power laws toward the same singular temperature $[4,5]$. The anomalies of the thermodynamic quantities become plausible if one postulates the existence of a second low-temperature critical point at about $228 \mathrm{~K}$ and at somewhat elevated pressure [6]. On the other hand, the transport coefficient anomalies are reminiscent of the dynamical behavior of a supercooled liquid near the socalled kinetic glass transition temperature, predicted by mode-coupling theory [7].

Search for the predicted [6] first-order liquid-liquid transition line and its end point, the second lowtemperature critical point $[1,8]$ in water, has been hampered by intervention of the homogenous nucleation process, which takes place at $235 \mathrm{~K}$ at the ambient pressure. However, by confining water in nanopores of mesoporous silica MCM-41-S with cylindrical pores of $14 \AA$ diameter, we have been able to study the dynamical behavior of water in a temperature range down to $160 \mathrm{~K}$, without crystallization. Using high-resolution quasielastic neutron scattering (QENS) method and relaxing-cage model (RCM) for the analysis, we determine the temperature and pressure dependences of the average translationalrelaxation time $\left\langle\tau_{T}\right\rangle$ for the confined supercooled water.

Micellar templated mesoporous silica matrices MCM41-S, which have 1D cylindrical pores arranged in 2D hexagonal arrays, were synthesized by following a similar method for synthesizing MCM-48-S previously [9]. Using the short chain cationic surfactants, $\mathrm{C}_{12} \mathrm{TMAB}$, as templates and adding $\beta$-type zeolite seeds as silica source [10], we obtain MCM-41-S ( $S$ denotes seed) with smaller pore sizes and stronger silica walls than the traditional MCM41. In this pressure experiment, we chose the silica with a pore diameter of $14 \AA$ because for the fully hydrated sample the differential scanning calorimetry data show no freezing peak down to $160 \mathrm{~K}$. The sample is then hydrated by exposing to water vapor in a closed chamber until it reaches the full hydration level of 0.5 gram $\mathrm{H}_{2} \mathrm{O} / 1$ gram silica.

High-resolution QENS spectroscopy method is used to determine the temperature and pressure dependences of $\left\langle\tau_{T}\right\rangle$ for the confined water. Because neutrons can easily penetrate the thick-wall high-pressure cell and because they are predominantly scattered by hydrogen atoms in water, rather than by the matrices containing it, incoherent QENS is an appropriate tool for our study. Using two separate high-resolution QENS spectrometers, we are able to measure the translational-relaxation time from 0.2 ps to 10000 ps over the temperature and pressure range. The high-pressure experiments were performed at both the high-flux backscattering (HFBS) and the disc-chopper time-of-flight (DCS) spectrometers in the NIST Center for Neutron Research (NIST NCNR). The two spectrometers used to measure the spectra have two widely different dynamic ranges (for the chosen experimental setup), one with an energy resolution of $0.8 \mu \mathrm{eV}$ (HFBS) and a dy- 
namic range of $\pm 11 \mu \mathrm{eV}$ [11], and the other with an energy resolution of $20 \mu \mathrm{eV}$ (DCS) and a dynamic range of $\pm 0.5 \mathrm{meV}$ [12] in order to be able to extract the broad range of relaxation times from the measured spectra. The same high-pressure system, including specially designed aluminum pressure cell, was used on the two instruments. Helium gas, the pressure-supplying medium, fills the whole sample cell, and applies pressure to the fully hydrated sample. The experiment at each pressure was done with a series of temperatures, covering both below and above the transition temperature. Altogether, 1000 spectra were collected, spanning 9 pressures: ambient, 100, 200, 400, 800, 1200, 1600, 2000, and 2400 bars.

QENS experiments measure the Fourier transform of the intermediate scattering function (ISF) of the hydrogen atoms, $F_{H}(Q, t)$, of water molecules in the pores of the silica matrix. Molecular dynamics (MD) simulations have shown that the ISF of both bulk [13] and confined [14] supercooled water can be accurately described as a twostep relaxation: a short-time Gaussian-like (in-cage vibrational) relaxation followed by a plateau and then a longtime (time $>1.0 \mathrm{ps}$ ) stretched exponential relaxation of the cage. The so-called relaxing-cage model (RCM) [15], which we use for data analysis, models closely this twostep relaxation and has been tested extensively against bulk and confined supercooled water through MD and experimental data [13-15]. By considering only the spectra with wave vector transfer $Q<1.1 \AA^{-1}$, we can safely neglect the contribution from the rotational motion of water molecule in ISF [15]. The RCM describes the translational dynamics of water at supercooled temperature in terms of the product of two functions:

$$
\begin{gathered}
F_{H}(Q, t) \approx F_{T}(Q, t)=F^{S}(Q, t) \exp \left\{-\left[t / \tau_{T}(Q)\right]^{\beta}\right\}, \\
\tau_{T}(Q)=\tau_{0}(0.5 Q)^{-\gamma}, \quad\left\langle\tau_{T}\right\rangle=\tau_{0} \Gamma(1 / \beta) / \beta,
\end{gathered}
$$

where the first factor, $F^{S}(Q, t)$, represents the short-time vibrational dynamics of the water molecule in the cage. This function is fairly insensitive to temperature variation, and thus can be calculated from MD simulation. The second factor, the $\alpha$-relaxation term, contains the stretch exponent $\beta$, and the $Q$-dependent translational-relaxation time $\tau_{T}(Q)$, which is a strong function of temperature. The latter quantity is further specified by two phenomenological parameters $\tau_{0}$ and $\gamma$, the exponent controlling the power law $Q$ dependence of $\tau_{T}(Q) .\left\langle\tau_{T}\right\rangle$ is a $Q$-independent quantity where $\Gamma$ is the gamma function. It essentially gives a measure of the structural relaxation time of the hydrogen-bond cage surrounding a typical water molecule. The temperature dependence of the translational-relaxation time at each pressure is then calculated from three fitted parameters, $\tau_{0}, \beta$, and $\gamma$, by analyzing a group of quasielastic peaks at different $Q$ values simultaneously. For this analysis, we chose seven spectra for data taken at HFBS and 11 spectra for data taken at DCS, at each temperature.
We show in Fig. 1, as an example, two complete sets (temperature series) of QENS area-normalized spectra. The broadening of the quasielastic peaks becomes more and more noticeable as temperature increases. In Fig. 1(a), we may notice, from shoulders of these spectral lines, that two groups of curves, 231-250 K and 198-209 K, are separated by the curve at a temperature of $220 \mathrm{~K}$. This visual information reinforces the result of the detail analysis shown in Fig. 2, that there is an abrupt dynamical transition at $T_{L}=216 \mathrm{~K}$. Figure 1(b) shows the RCM analysis of the spectrum taken at $T=220 \mathrm{~K}$, close to the transition temperature. On the other hand, in Fig. 1(c), the spectra at pressure 1600 bar show a rather smooth variation with temperature, indicating that there is no sharp transition. This pressure corresponds to the end point of the line of FS transitions shown in Fig. 3. Figure 1(d) is, again, an RCM analysis of the spectrum taken at $T=214 \mathrm{~K}$ of this pressure. RCM, as one can see, reproduces the experimental spectral line shapes of confined water well. The broadening of the experimental data over the resolution function, shown in Figs. 1(b) and 1(d) leaves enough dynamic information to be extracted by RCM.

The behavior of shear viscosity $\eta$ or equivalently the structural relaxation time $\tau$ of a supercooled liquid approaching its glass transition temperature is called "fragile" when it varies according to the so-called VogelFulcher-Tammann (VFT) law, and the behavior is called "strong" when $\eta$ or $\tau$ obeys Arrhenius law [16]. For water, a fragile liquid at room temperature and at moderately supercooled temperatures, Ito and co-workers [17] proposed that a "fragile-to-strong" transition would occur at around $228 \mathrm{~K}$, based on a thermodynamic argument. The FS transition in a molecular liquid like water may be interpreted as a variant of kinetic glass transition predicted

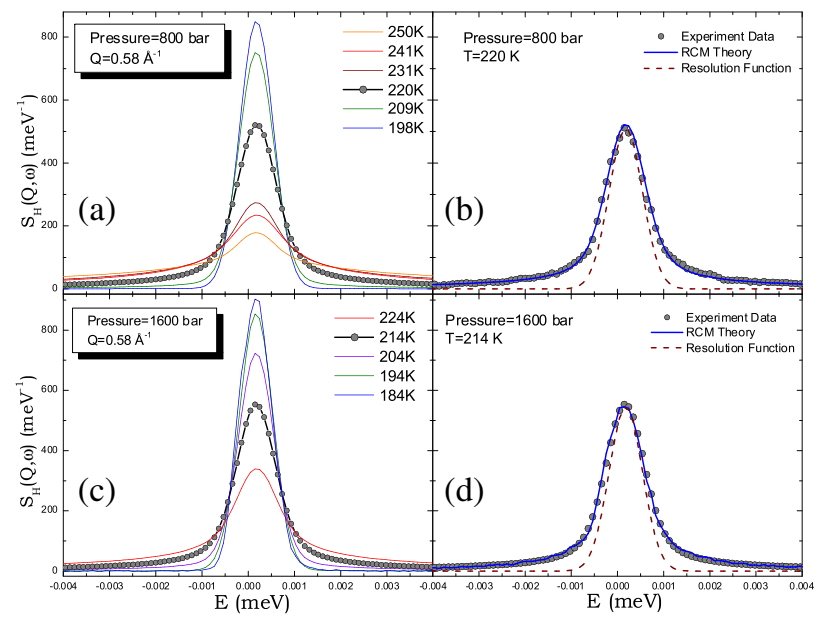

FIG. 1 (color online). (a) and (c) (left panels) show QENS spectra measured at $Q=0.58 \AA^{-1}$, at two pressures, $800 \mathrm{bar}$ and 1600 bar, and at a series of temperatures. (b) and (d) (right panels) show the RCM analysis of one of the spectra from each pressure. The resolution function in each case is shown by a dashed line. 

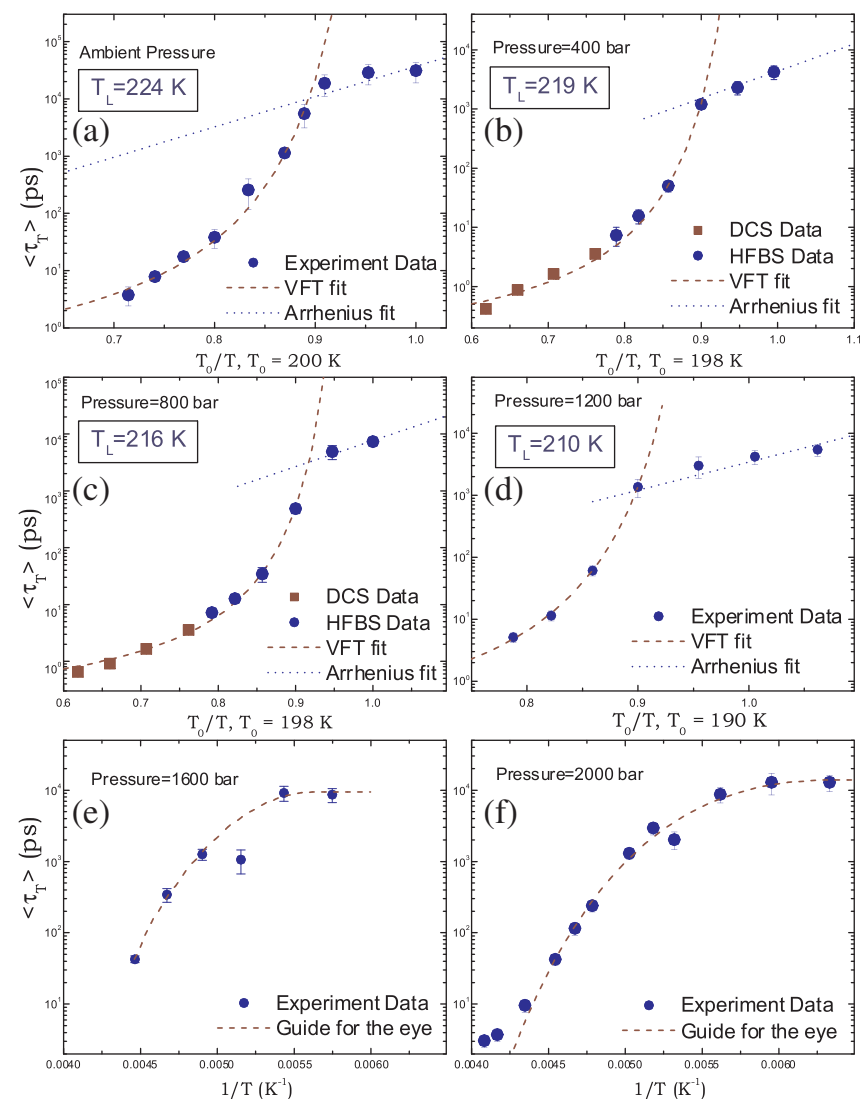

FIG. 2 (color online). Temperature dependence of $\left\langle\tau_{T}\right\rangle$ plotted in $\log \left(\left\langle\tau_{T}\right\rangle\right)$ vs $T_{0} / T$ or $1 / T$. Data from ambient pressure, 400, $800,1200,1600$, and 2000 bars are shown in panels (a), (b), (c), (d), (e), and (f), respectively.

by the ideal mode-coupling theory [7], where the real structural arrest transition is avoided by an activated hopping mechanism below the transition.

In Fig. 2, we report the temperature variation of $\left\langle\tau_{T}\right\rangle$ for water molecules as a function of pressure. It is seen that Figs. 2(a)-2(d) show clearly a transition from a VFT law: $\left\langle\tau_{T}\right\rangle=\tau_{1} \exp \left[D T_{0} /\left(T-T_{0}\right)\right]$, where $D$ is a constant providing the measure of fragility and $T_{0}$, the ideal glass transition temperature, to an Arrhenius law: $\left\langle\tau_{T}\right\rangle=$ $\tau_{1} \exp \left[E_{A} / R T\right]$, where $E_{A}$ is the activation energy for the relaxation process and $R$ the gas constant. This transition of VFT to Arrhenius behavior, previously observed at ambient pressure [18], is the signature of a FS dynamic transition predicted by Ito et al. [17]. In this Letter, we show the extension of this transition into finite pressures. The transition temperature, $T_{L}$, as the crossing point of the VFT law and Arrhenius law, is calculated by $1 / T_{L}=1 / T_{0}-$ $\left(D k_{B}\right) / E_{A}$. However, in Figs. 2(e) and 2(f), the cusplike transition becomes rounded off and there is no clear-cut way of defining the FS transition temperature. Note that in Fig. 2(f), there is still a hint of fragile behavior at high enough temperature.

Summarizing all the results, we show in a $P-T$ plane, in Fig. 3, the observed pressure dependence of $T_{L}$ and its estimated continuation, denoted by a dashed line, in the

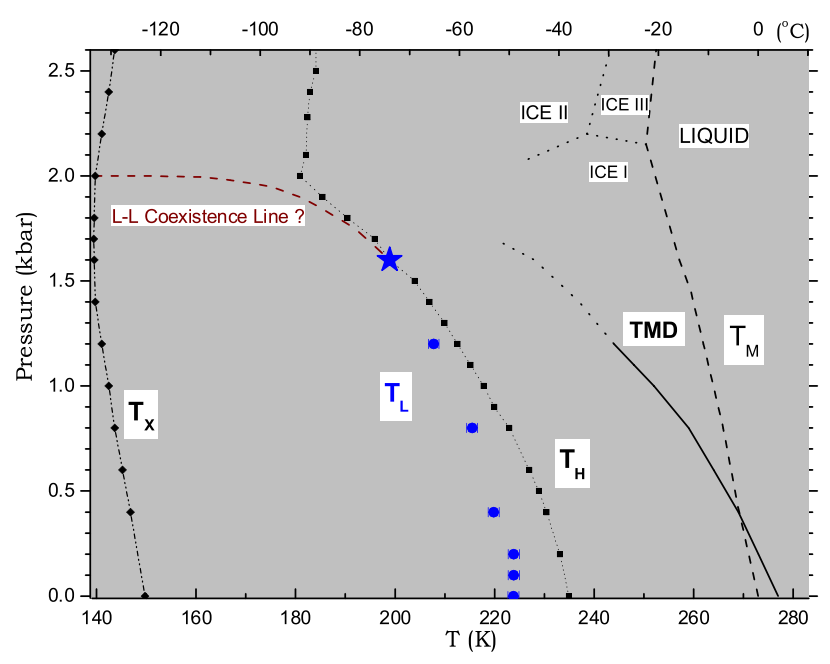

FIG. 3 (color online). The pressure dependence of the FS dynamic transition temperature, $T_{L}$, plotted in the $P-T$ plane (solid circles). Also shown are the homogeneous nucleation temperature line, denoted as $T_{H}$ [25], crystallization temperatures of amorphous solid water [26], denoted as $T_{X}$, and the temperature of maximum density line, denoted as TMD [27].

pressure region where no clear-cut FS transition is observed. One should note that the $T_{L}$ line has a negative slope, parallel to TMD line, indicating a lower density liquid on the lower temperature side. This $T_{L}$ line also approximately tracks the $T_{H}$ line, and terminates in the upper end when intersecting the $T_{H}$ line at 1600 bar and $200 \mathrm{~K}$, at which point the character of the dynamic transition changes. We shall discuss the significance of this point later on. A special feature of the $T_{L}$ line at the lower end should be noted as well. The line essentially becomes vertical after around 200 bar and the transition temperature approaches a constant value of $\sim 225 \mathrm{~K}$.

Since $T_{L}$ determined experimentally is a dynamic transition temperature, it is natural to question whether the system is in a liquid state on both sides of the $T_{L}$, and if so, what would the nature of the high-temperature and lowtemperature liquids be? Sastry and Angell have recently shown by a MD simulation that at a temperature $T \approx$ $1060 \mathrm{~K}$ (at zero pressure), below the freezing point $1685 \mathrm{~K}$, the supercooled liquid silicon undergoes a firstorder liquid-liquid phase transition, from a fragile, dense liquid to a strong, low-density liquid with nearly tetrahedral local coordination [19]. Prompted by this finding, we may like to relate, in some way, our observed $T_{L}$ line to the liquid-liquid transition line, predicted by MD simulations of water [6] and speculating on the possible location of the low-temperature critical point.

According to our separate inelastic neutron scattering experiments, which measure the librational density of states of water contained in $18 \AA$ pore size MCM-41-S, water remains in disordered liquid state both above and below the FS transition at ambient pressure. Furthermore, our analysis of the FS transition for the case of ambient pressure indicates that the activation energy bar- 
rier for initiating the local structural relaxation is $E_{A}=$ $4.89 \mathrm{Kcal} / \mathrm{mol}$ for the low-temperature strong liquid. Yet previous inelastic scattering experiments of stretch vibrational band of water [20] indicate that the effective activation energy of breaking a hydrogen bond at $258 \mathrm{~K}$ (hightemperature fragile liquid) is $3.2 \mathrm{Kcal} / \mathrm{mol}$. Therefore, it is reasonable to conclude that the high-temperature liquid corresponds to the high-density liquid (HDL) where the locally tetrahedrally coordinated hydrogen-bond network is not fully developed, while the low-temperature liquid corresponds to the low-density liquid (LDL) where the more open, locally icelike hydrogen-bond network is fully developed [21].

It is appropriate now to address the possible location of the second critical point [6]. Above the critical temperature $T_{C}$ and below the critical pressure $p_{C}$, we expect to find a one-phase liquid with a density $\rho$, which is constrained to satisfy an equation of state: $\rho=f(p, T)$. If an experiment is done by varying temperature $T$ at a constant pressure $p<p_{C}, \rho$ will change from a high-density value (corresponding to HDL) at sufficiently high temperature to a low-density value (corresponding to LDL) at sufficiently low temperature. Since the fragile behavior is associated with HDL and the strong behavior with LDL, we should expect to see a clear FS transition as we lower the temperature at this constant $p$. Therefore, the cusplike FS transition we observed should then occur when we cross the so-called Widom line in the one-phase region $[22,23]$. On the other hand, if the experiment is performed in a pressure range $p>p_{C}$, corresponding to the two-phase region and crossing the liquid-liquid $(L-L)$ coexistence line, the system will consist of mixture of different proportions of HDL and LDL as one varies $T$. In this latter case, $\left\langle\tau_{T}\right\rangle$ versus $1 / T$ plot will not show a clear-cut FS transition (the transition will be washed out) because the system is in a mixed state. The above picture would then explain the dynamical behavior we showed in Fig. 2. In Fig. 2, a clear FS transition is observed up to 1200 bar and beyond 1600 bar the transition is rounded off. From this observation, the reasonable location of the $L-L$ critical point is estimated to be at $p_{C}=1600 \pm 400 \mathrm{bar}$ and $T_{C}=$ $200 \pm 10 \mathrm{~K}$, shown by a star in Fig. 3.

Additionally, in a recent MD simulation using 3 different waterlike potentials [23], a small peak was found in the specific heat $C_{P}$ when crossing the Widom line at a constant $p$. Meanwhile, Maruyama et al. conducted an experiment on adiabatic calorimetry of water confined within nanopores of silica gel [24]. It was found that water within $30 \AA$ pores was well prevented from crystallization, and also showed a small $C_{P}$ peak at $227 \mathrm{~K}$ at ambient pressure. This experimental result further supports that the FS transition we observed at $225 \mathrm{~K}$ at ambient pressure is caused by the crossing of the Widom line in the one-phase region above the critical point [23].

The research at MIT is supported by DOE Grants No. DE-FG02-90ER45429 and No. 2113-MIT-DOE-591. This work utilized facilities supported in part by the
National Science Foundation under Agreement No. DMR-0086210. This work was also supported by the National Science Council of Taiwan. Technical supports in measurements from E. Mamontov, J. Leao, and J.R.D. Copley at NIST NCNR are greatly appreciated. We acknowledge an enlightening discussion with C. A. Angell on this subject. We benefited from affiliation with EU-MariCurie Research and Training Network on Arrested Matter.

*Author to whom correspondence should be addressed. Electronic address: sowhsin@mit.edu

[1] P. G. Debenedetti and H. E. Stanley, Phys. Today 56, No. 6, 40 (2003).

[2] R. J. Speedy and C. A. Angell, J. Chem. Phys. 65, 851 (1976).

[3] C. A. Angell et al., J. Phys. Chem. 77, 3092 (1973).

[4] F. X. Prielmeier et al., Phys. Rev. Lett. 59, 1128 (1987).

[5] Yu. A. Osipov et al., Russ. J. Phys. Chem. 51, 1264 (1977).

[6] P. H. Poole et al., Nature (London) 360, 324 (1992).

[7] W. Götze et al., Rep. Prog. Phys. 55, 241 (1992). However, see also an alternative theory by J.P. Garrahan and D. Chandler, Proc. Natl. Acad. Sci. U.S.A. 100, 9710 (2003).

[8] P. G. Debenedetti, J. Phys. Condens. Matter 15, R1669 (2003).

[9] P. C. Shih et al., Stud. Surf. Sci. Catal. 146, 557 (2003).

[10] Y. Liu et al., J. Am. Chem. Soc. 122, 8791 (2000).

[11] A. Meyer et al., Rev. Sci. Instrum. 74, 2759 (2003).

[12] J.R. D. Copley and J.C. Cook, Chem. Phys. 292, 477 (2003).

[13] P. Gallo et al., Phys. Rev. Lett. 76, 2730 (1996).

[14] P. Gallo et al., Phys. Rev. Lett. 85, 4317 (2000).

[15] S.-H. Chen et al., Phys. Rev. E 59, 6708 (1999).

[16] C. A. Angell, J. Non-Cryst. Solids 131-133, 13 (1991).

[17] K. Ito et al., Nature (London) 398, 492 (1999).

[18] A. Faraone et al., J. Chem. Phys. 121, 10843 (2004).

[19] S. Sastry and C. A. Angell, Nat. Mater. 2, 739 (2003).

[20] M. A. Ricci and S.-H. Chen, Phys. Rev. A 34, 1714 (1986).

[21] A. K. Soper et al., Phys. Rev. Lett. 84, 2881 (2000).

[22] L. Xu, S. Buldyrev, F. Sciortino, P. Kumar, and H.E. Stanley (private communication). According to these researchers, the Widom line is defined as a line in the $P-T$ plane, starting from the critical point $\left(p_{C}, T_{C}\right)$ and extending into the one-phase region, with the same slope as that of the $L-L$ coexistence line at $\left(p_{C}, T_{C}\right)$. This line is an imaginary line where many thermodynamic quantities and transport coefficients show a peak when crossing it.

[23] L. Xu, P. Kumar, S. V. Buldyrev, S.-H. Chen, P. H. Poole, F. Sciortino, and H.E. Stanley, Proc. Natl. Acad. Sci. U.S.A., "Relation between the Widom Line and a Dynamic Crossover: Evidence of a Liquid-Liquid Critical Point," (in press).

[24] S. Maruyama et al., AIP Conf. Proc. 708, 675 (2004).

[25] H. Kanno et al., Science 189, 880 (1975).

[26] H.E. Stanley, Mysteries of Water, edited by M.-C. Bellissent-Funel, Nato Science Series A Vol. 305 (IOS Press, Amsterdam, 1999).

[27] C. A. Angell et al., J. Non-Cryst. Solids 205-207, 463 (1996). 